\title{
Determining graphene's induced band gap with magnetic and electric emitters
}

\author{
Julia F. M. Werra, ${ }^{1, *}$ Peter Krüger, ${ }^{2}$ Kurt Busch, ${ }^{1,3}$ and Francesco Intravaia ${ }^{3}$ \\ ${ }^{1}$ Humboldt-Universität zu Berlin, Institut für Physik, AG Theoretische Optik \& Photonik, Newtonstraße 15, 12489 Berlin, Germany \\ ${ }^{2}$ Midlands Ultracold Atom Research Centre, School of Physics and Astronomy, University of Nottingham, \\ Nottingham NG7 2RD, United Kingdom \\ ${ }^{3}$ Max-Born-Institut, Max-Born-Straße 2A, 12489 Berlin, Germany
}

(Received 13 November 2015; published 9 February 2016)

\begin{abstract}
We present numerical and analytical results for the lifetime of emitters in close proximity to graphene sheets. Specifically, we analyze the contributions from different physical channels that participate in the decay processes. Our results demonstrate that measuring the emitters' decay rates provides an efficient route for sensing graphene's optoelectronic properties, notably the existence and size of a potential band gap in its electronic band structure.
\end{abstract}

DOI: 10.1103/PhysRevB.93.081404

Driven by its successful isolation, graphene has not stopped fascinating the research community. Although this allotropic form of carbon had been theoretically investigated for decades, experimental access to graphene has offered new perspectives as well as novel directions for fundamental research and technological applications [1,2]. Graphene's exotic properties [3] have led to the investigation of a wide range of phenomena such as ballistic transport [4], the quantum Hall effect [1,5], and thermal [6] as well as electrical conductivity [7,8]. Developing a detailed understanding, followed by appropriate engineering, of these properties lies at the heart of future graphene-based technologies. For this, an accurate determination of graphene's properties in realistic experimental settings and the detailed validation of various theoretical models (cf. Ref. [7,9-11]) is indispensable. Promising designs where the semimetal will play an important role aim at combining condensed matter with atomic systems. Such hybrid devices are geared towards reaping the best of the two worlds for advanced high-performance devices.

In this work, we demonstrate how the high degree of control and accuracy available in quantum systems like cold atoms and $\mathrm{Si}$ and NV centers in nanodiamonds can be employed for detailed investigations of graphene's optoelectronic properties [12-15]. Specifically, we focus on modifications in the lifetimes of emitters held in close proximity to graphene layers and show that these allow for direct experimental access to features like band gaps as well as plasmons and/or plasmonlike resonances. In graphene, a band gap $\Delta$ (cf. Fig. 1) is created (i) when the atomically thin material is deposited on a substrate [16,17], (ii) when strain is applied, (iii) when impurities are present, and (iv) in cases where graphene bilayers instead of a single layer are considered. Values for $\Delta$ of the order of tens of meV have been predicted $[16,17]$, thus triggering corresponding experimental investigations. These band gaps and the features connected with them are still the subject of discussions $[18,19]$ so that reliable experimental means for their analysis are highly desirable.

For planar geometries the decay rate of an emitter is a functional of the system's optical scattering coefficients. We model

\footnotetext{
*jwerra@physik.hu-berlin.de
}

a monoatomic graphene layer in terms of a $2+1$-dimensional Dirac fluid [10,20,21] and embed it in a nondispersive and nondissipative dielectric medium with permittivity $\varepsilon_{m}$. As a result, the graphene layer is characterized by an induced band gap and a chemical potential $\mu=0$ (cf. Fig. 1) while the corresponding electromagnetic reflection coefficients for transverse magnetic (TM) and transverse electric (TE) waves are $[20,21]$

$$
r^{\mathrm{TM}}=-\frac{\alpha \Phi(y)}{y \varepsilon_{m} / \kappa_{m}-\alpha \Phi(y)}, r^{\mathrm{TE}}=-\frac{\alpha \Phi(y)}{\kappa_{m}+\alpha \Phi(y)},
$$

where $\alpha=137^{-1}$ is the fine structure constant and

$$
\Phi(y)=1-(\sqrt{y}+1 / \sqrt{y}) \operatorname{arctanh}(\sqrt{y}),
$$

with $y=\omega^{2}-v_{\mathrm{F}}^{2} k^{2}$. Further, $\kappa_{m}=\sqrt{k^{2}-\varepsilon_{m} \omega^{2}}$ and $k=$ $\sqrt{k_{x}^{2}+k_{y}^{2}}$ denote, respectively, the moduli of the out-of-plane and in-plane wave vectors in the dielectric medium. In addition, we use dimensionless variables, which amounts to the replacements $\hbar \omega / 2 \Delta \rightarrow \omega, \hbar c k / 2 \Delta \rightarrow k$, and $v_{\mathrm{F}} / c \rightarrow v_{\mathrm{F}}$ $\left(\approx 300^{-1}\right.$ for graphene). Lifetime modifications are usually associated with the strength of scattering processes. Owing to its minute thickness (few $\AA$ ), the optical response of a single graphene layer is rather small ( $\sim 2 \%$ reflection [22]). Thus, for emitters near a graphene layer, small lifetime modifications might naively be expected. However, graphene's exotic properties introduce additional features that affect the

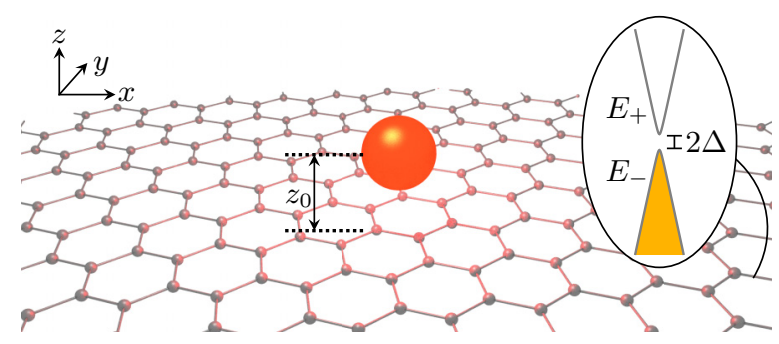

FIG. 1. Schematic of the physical situation considered in this work. An emitter (red sphere) is positioned at distance $z_{0}$ from a graphene sheet. Graphene's band structure is approximated by $E_{ \pm}=$ $\pm \sqrt{\Delta^{2}+v_{\mathrm{F}}^{2} k^{2}}$ (see inset) and the chemical potential is chosen as $\mu=0$ (yellow: filled band). 


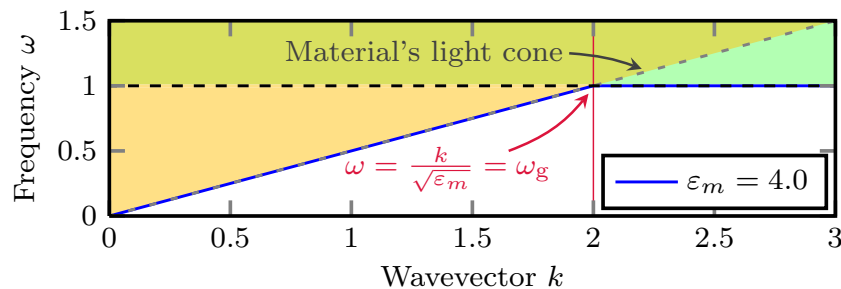

FIG. 2. Dispersion relation of the TE surface plasmon (blue line) for a graphene layer embedded in a dispersionless dielectric material $\left(\varepsilon_{m}=4.0\right)$. The colored areas delineate the wave-vector regions corresponding to different decay channels: propagating waves (yellow), evanescent waves (white), and single-particle excitations (green).

emitters' dynamics, such as TE plasmons and single (SPE)and multiple (MPE)-particle excitations.

Different frequencies are associated with the different physical processes: propagating fields occur for $0 \leqslant k<\omega \sqrt{\varepsilon_{m}}$ and evanescent fields are characterized by $k>\omega \sqrt{\varepsilon_{m}}$. Further, we identify another regime where $k<\sqrt{\omega^{2}-1} / v_{\mathrm{F}}$, which only exists if $\omega>1$, i.e., if the radiation frequency exceeds that associated with the band gap. In this regime, the $2+1-$ dimensional Dirac fluid model of graphene features the creation of electron-hole pairs. In the propagating regime, the scattering process in graphene systems is very similar to that in ordinary thin films. This similarity, however, already breaks down for evanescent waves, for which the scattering process is associated with surface plasmons or plasmonlike phenomena: While in ordinary materials these resonances are usually present only in TM polarization, graphene is known for admitting such excitations in both TM and TE polarization $[10,11,23,24]$. TM-polarized surface plasmons are associated with charge density oscillations and are dominated by the electric field. Conversely, TE plasmons result from resonances in the motion of the current density so that they are dominated by the magnetic field. Mathematically, these phenomena are related to divergences of the scattering coefficients and in our case they can be investigated by analyzing the poles of Eq. (1). In our model TM plasmons do not occur, while the TE plasmon's dispersion relation reads as

$$
\left\{\begin{array}{l}
\omega[y]=\sqrt{y+v_{\mathrm{F}}^{2} k[y]^{2}} \\
k[y]=\omega_{\mathrm{g}} \sqrt{\alpha^{2} \Phi(y)^{2}+\varepsilon_{m} y},
\end{array}\right.
$$

where $\omega_{\mathrm{g}}=1 / \sqrt{1-\varepsilon_{m} v_{\mathrm{F}}^{2}}$. This agrees well with previous numerical results for vacuum $\left(\varepsilon_{m}=1\right)$ [10].

Albeit difficult to discern in Fig. 2, Eq. (3) indicates that the TE plasmon's dispersion relation lies exclusively in the evanescent region and stays outside of the single-particle excitation region (SPE) [25]. Two distinct characteristics become apparent: For low frequencies $\left(\omega<\omega_{\mathrm{g}}\right)$, the dispersion curve lies close to but below the medium's light cone; for large frequencies $\left(\omega>\omega_{\mathrm{g}}\right)$, the properties of the TE plasmon's dispersion do not depend on the embedding dielectric but are solely determined by graphene itself.

With respect to the processes described above, the total decay rate of an emitter with dipole operator $\hat{\mathbf{d}}$ can be written as $\gamma / \gamma_{0}=1+\mathcal{L}\left[\left(d_{\|}^{2} /|\mathbf{d}|^{2}\right) \Gamma^{\|}+\left(d_{\perp}^{2} /|\mathbf{d}|^{2}\right) \Gamma^{\perp}\right]$, where $\gamma_{0}$ is the decay rate in a homogeneous dielectric without graphene. The factor $\mathcal{L}$ indicates the usually frequency-dependent local field correction one has to take into account to correctly describe the dynamics of an emitter embedded in a dielectric $\left(\mathcal{L}=1\right.$ for $\epsilon_{m}=1$ ) [26,27]. For simplicity, we will not dwell on this issue and instead refer readers to the literature for further information [26-29]. The functions $\Gamma^{\|, \perp}$ are related to the matrix elements of the orthogonal $d_{\perp}$ and parallel $d_{\|}$ components of the dipole with respect to the graphene layer $\left(|\mathbf{d}|^{2}=d_{\|}^{2}+d_{\|}^{2}\right)$. In turn, each of these two contributions is the result of the three processes discussed above. Consequently, we have the radiative term $\Gamma_{\mathrm{r}}$, which originates from the propagating region (including the radiative part of the SPE region), the contribution of the (nonradiative) SPE region $\Gamma_{\mathrm{SPE}}$, and the nonradiative contribution given by plasmonic excitations $\Gamma_{\mathrm{p}}$.

In order to analyze the above terms in more detail, we will first discuss the case of magnetic decay, keeping in mind that a magnetic emitter ought to be more sensitive to the magnetic field associated with plasmonic TE resonances. The emitter has a transition frequency $\omega_{0}$ and is located at $z=z_{0}>0$ above the graphene layer at $z=0$ (see Fig. 1). Within second-order perturbation theory $[30,31]$ the modification of the decay rate can be written as

$$
\begin{gathered}
\Gamma^{\|}=\frac{3}{4} \int_{-\infty}^{\omega_{0}^{2}} d y \operatorname{Im}\left[\frac{\frac{r^{\mathrm{TM}}}{K_{s}[y]}+\frac{K_{s}[y] r^{\mathrm{TE}}}{k_{0}^{2} v_{\mathrm{F}}^{2}}}{2 k_{0} v_{\mathrm{F}}} \exp \left(-2 d \frac{K_{s}[y]}{v_{\mathrm{F}}}\right)\right], \\
\Gamma^{\perp}=\frac{3}{2} \int_{-\infty}^{\omega_{0}^{2}} d y \operatorname{Im}\left[\frac{k_{s}[y]^{2}}{K_{s}[y]} \frac{r^{\mathrm{TE}}}{2 k_{0}^{3} v_{\mathrm{F}}^{3}} \exp \left(-2 d \frac{K_{s}[y]}{v_{\mathrm{F}}}\right)\right] .
\end{gathered}
$$

Here, $k_{0}=\omega_{0} \sqrt{\varepsilon_{m}}, d=2 z_{0} \Delta / \hbar c$. We have also defined $k_{s}[y]=\sqrt{\omega_{0}^{2}-y}$ and $K_{s}[y] \equiv v_{\mathrm{F}} \kappa[y]=\sqrt{\omega_{0}^{2} / \omega_{\mathrm{g}}^{2}-y}$. In Eqs. (4), the evanescent contribution is associated with the range $-\infty \leqslant y \leqslant\left(\omega_{0} / \omega_{\mathrm{g}}\right)^{2}$, while the $\left(\omega_{0} / \omega_{\mathrm{g}}\right)^{2} \leqslant y \leqslant$ $\omega_{0}^{2}(<1)$ corresponds to the propagating region. The SPE range corresponds to $1<y<\omega_{0}^{2}$.

We first consider the contribution to the decay rate from the evanescent range, imputable only to the resonance in the reflection coefficients. In view of the above discussion of the dispersion relation, Eq. (3), this contribution features two different regimes. For $\omega_{0}<\omega_{\mathrm{g}}$, i.e., when the dispersion curve is very close to the light cone, the resonance is located at $y_{\mathrm{p}} \approx\left(\omega_{0} / \omega_{\mathrm{g}}\right)^{2}\left[1-\left(4 \alpha v_{\mathrm{F}} / 3\right)^{2}\left(\omega_{0} / \omega_{\mathrm{g}}\right)^{2}\right]$. The leading terms of Eq. (4) are then

$$
\Gamma_{\mathrm{p}}^{\|} \approx \frac{16 \alpha^{3} \pi}{9 \varepsilon_{m}^{3 / 2}} \frac{\omega_{0}^{3}}{\omega_{\mathrm{g}}^{3}} \exp \left(-d / d_{0}\right), \Gamma_{\mathrm{p}}^{\perp} \approx \frac{2 \pi \alpha}{\sqrt{\varepsilon_{m}}} \frac{\omega_{0}}{\omega_{\mathrm{g}}^{2}} \exp \left(-d / d_{0}\right) .
$$

Given the rather large characteristic decay length $d_{0}=$ $\left[3 \epsilon_{m} \omega_{\mathrm{g}}^{2} /(8 \alpha)\right] k_{0}^{-2}$, these contributions exhibit weak distance dependencies for experimentally relevant emitter-graphene separations of a few microns. For $\omega_{0}>\omega_{\mathrm{g}}$, the resonance is instead located close to the boundary of the SPE region, 

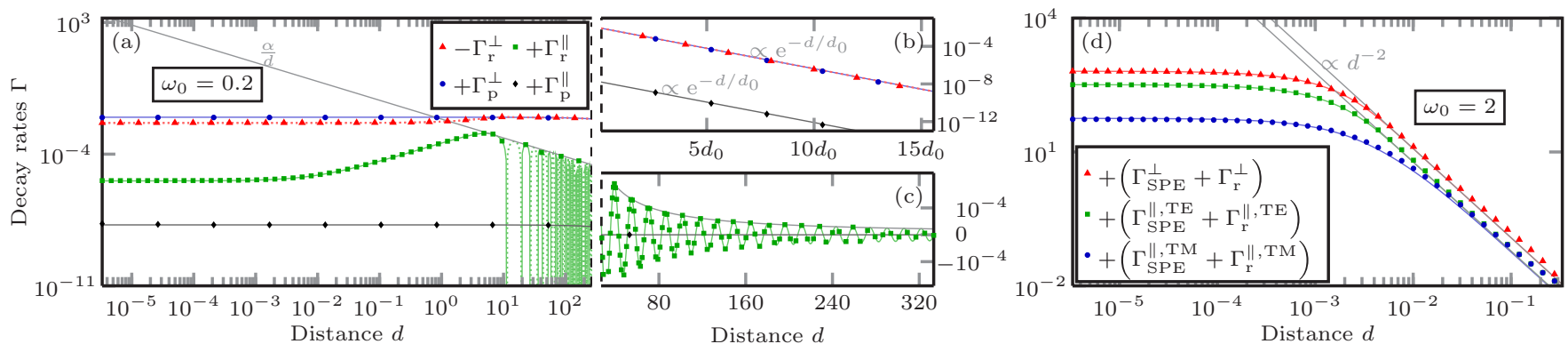

FIG. 3. Distance behavior of the different contributions to a magnetic emitter's decay rate for an emitter above a free-standing graphene layer $\left(\epsilon_{m}=1\right)$. Panels (a)-(c) and (d) display results for transistion frequencies well below $\left(\omega_{0}=0.2\right)$ and well above $\left(\omega_{0}=2.0\right)$ the band gap of graphene, respectively. Symbols correspond to complete numerical solutions and lines to approximate analytical solutions (see text for details). Panels (b) and (c) represent continuations of the $\mathrm{x}$ axis shown in panel (a), and panel (c) is plotted on a linear scale. Note that the results are plotted on a logarithmic scale so that contributions leading to an enhancement $(+)$ or a suppression (-) of the decay rate are indicated by corresponding signs in the inset of panels (a) and (d). Further, for distances $d>10$ the (for small distances strictly positive) contributions from $\Gamma_{r}^{\|}$oscillate around zero as depicted in panel (c).

$y_{\mathrm{p}} \approx 1-2 \exp \left\{-\left[1+K_{s}[1] /\left(\alpha v_{\mathrm{F}}\right)\right]\right\}$ and we obtain

$$
\begin{gathered}
\Gamma_{\mathrm{p}}^{\|} \approx \frac{3 \pi K_{s}[1] \mathrm{e}^{-\left(1+\frac{K_{s}[1]}{\alpha v_{\mathrm{F}}}\right)}}{2 \alpha v_{\mathrm{F}}^{4} k_{0}^{3}} \exp \left(-2 d \frac{K_{s}[1]}{v_{\mathrm{F}}}\right), \\
\Gamma_{\mathrm{p}}^{\perp} \approx \frac{3 \pi k_{s}[1] \mathrm{e}^{-\left(1+\frac{K_{s}[1]}{\alpha v_{\mathrm{F}}}\right)}}{\alpha v_{\mathrm{F}}^{4} k_{0}^{3}} \exp \left(-2 d \frac{K_{s}[1]}{v_{\mathrm{F}}}\right) .
\end{gathered}
$$

Due to the small values of $v_{\mathrm{F}}$ and $\alpha$, the above terms are strongly suppressed in graphene unless $K_{s}[1] \sim 0$, which only occurs when $\omega_{0} \sim \omega_{\mathrm{g}} \gtrsim 1$.

For the same parameters, the propagating regime corresponds to a rather small integration range in Eq. (4). Therefore, the integrands can be expanded around $y=\omega_{0}^{2}$ and after some rearrangements we obtain

$$
\begin{aligned}
& \Gamma_{\mathrm{r}}^{\|} \approx \frac{\alpha\left(\varepsilon_{m}+1\right)}{2 \varepsilon_{m} d}\left[\frac{4 \alpha k_{0}\left(\varepsilon_{m}+3\right)}{9 \varepsilon_{m}\left(\varepsilon_{m}+1\right)} \sin \left(2 k_{0} d\right)\right. \\
&\left.+\frac{\sin \left(2 k_{0} d\right)}{2 k_{0} d}-\cos \left(2 k_{0} d\right)\right] \\
& \Gamma_{\mathrm{r}}^{\perp} \approx-\frac{\omega_{g}^{2} d}{2 d_{0}} \int_{0}^{2 k_{0} d} d \zeta \frac{\left[1-\left(\frac{\zeta}{2 k_{0} d}\right)^{2}\right]\left[\frac{\zeta \sin (\zeta)}{2 k_{0} d}+\frac{\omega_{g}^{2} \cos (\zeta)}{2 k_{0} d_{0}}\right]}{\left(\frac{\omega_{g}^{2} d}{d_{0}}\right)^{2}+\zeta^{2}} \\
& \stackrel{d \ll k_{0}^{-1}}{\approx}-\frac{\pi \alpha}{\sqrt{\varepsilon_{m}}} \omega_{0}\left(1+\frac{8 k_{0} d}{3 \pi}\right) .
\end{aligned}
$$

Interestingly, because of the overall minus sign of $\Gamma_{r}^{\perp}$, this contribution tends to increase the emitter's lifetime, suppressing the decay process relative to $\gamma_{0}$. In addition, since $k_{0}^{-1} \ll$ $d_{0} / \omega_{g}^{2}$, due to the dephasing between the propagating waves, $\Gamma_{\mathrm{r}}^{\perp}$ exponentially decays for distances $d \gtrsim d_{0} / \omega_{g}^{2}$. It follows a behavior similar to the TE plasmon but with characteristic decay length $d_{0} / \omega_{g}^{2}$. Therefore, since $\omega_{g} \sim 1, \Gamma_{\mathrm{r}}^{\perp}$ is almost exactly canceled by $\Gamma_{\mathrm{p}}^{\perp}$ [see Fig. 3(b)]. For even larger distances $\left(d \gg d_{0} / \omega_{g}^{2}\right.$, not shown), due to the interference between incoming and scattered waves, $\Gamma_{\mathrm{r}}^{\perp}$ oscillates in space like $\Gamma_{\mathrm{r}}^{\|}$with a frequency $2 k_{0}$ [see Fig. 3(c)].
Finally, we consider the modification of the decay rate that stems from the SPE region. This contribution only occurs when the emitter's transition frequency becomes larger than the electronic band gap $\left(\omega_{0}>1\right)$. Although the total SPE region includes both evanescent and propagating contributions, the nonradiative part dominates at short distances and, as in the previous case, is almost constant for $d \ll k_{0}^{-1}$. Again, since $\alpha, v_{\mathrm{F}} \ll 1$, in this limit we can write

$$
\Gamma_{\mathrm{SPE}}^{\perp} \approx \frac{\alpha \pi}{4 v_{\mathrm{F}}^{2} \varepsilon_{1}^{3 / 2} \omega_{\mathrm{g}}^{3}}\left[1+3\left(\frac{\omega_{\mathrm{g}}}{\omega_{0}}\right)^{2}-4\left(\frac{\omega_{\mathrm{g}}}{\omega_{0}}\right)^{3}\right] .
$$

This demonstrates that $\Gamma_{\text {SPE }}^{\perp}$ varies nonmonotonously with frequency and exhibits a maximum for $\omega_{0}=2 \omega_{\mathrm{g}}$, where it takes the value $\Gamma_{\mathrm{SPE}}^{\perp} \approx 645$. At intermediate distances, the total (evanescent and propagating) SPE contribution decays as a power law, $\Gamma_{\mathrm{SPE}}^{\perp}+\Gamma_{\mathrm{r}}^{\perp} \approx 2\left(\Gamma_{\mathrm{SPE}}^{\|}+\Gamma_{\mathrm{r}}^{\|}\right) \approx \alpha \pi \omega_{\mathrm{g}}\left(\omega_{0}^{-2}+\right.$ 1) $\left(6 \omega_{0} d\right)^{-2}$ [see Fig. 3(d)]. For $d \gg k_{0}^{-1}$, the propagating waves induce once again spatial oscillations with frequency $2 k_{0}$ (not shown).

In Fig. 4(a) we present the frequency dependence of all the above-discussed contributions to the decay rate at a fixed distance $d=\left(3 \times 10^{8}\right)^{-1}$ from the graphene layer (corresponding to $z_{0}=1 \mu \mathrm{m}$ for an emitter with transition frequency of $1 \mathrm{MHz}$ ). As discussed above, for emitters with transition frequencies smaller than graphene's electronic band gap $\left(\omega_{0}<1\right)$, the two main decay channels are the TE plasmonic resonance and the radiative decay. Their relative importance differs, depending on the spatial orientation of the dipole-matrix elements. We see that in $\Gamma^{\perp}$ the plasmonic TE resonance provides an enhancement while the radiative contribution leads to a suppression. Also, $\Gamma_{\mathrm{p}}^{\perp} \approx-2 \Gamma_{\mathrm{r}}^{\perp}$ over a very large range of frequencies. For $\Gamma^{\|}$, the radiative contribution dominates and leads to an enhancement of the decay rate. In this case, the plasmonic TE resonance, due to its proportionality to $\omega_{0}^{3}$, represents a subleading contribution. For $\omega_{0}>1$, the dominant contribution for both $\Gamma^{\perp}$ and $\Gamma^{\|}$ stems from the SPE contribution [see Fig. 4(a), inset] and leads to an enhancement of the decay rate by three orders of magnitude. Note that the increase of the decay rate occurs 

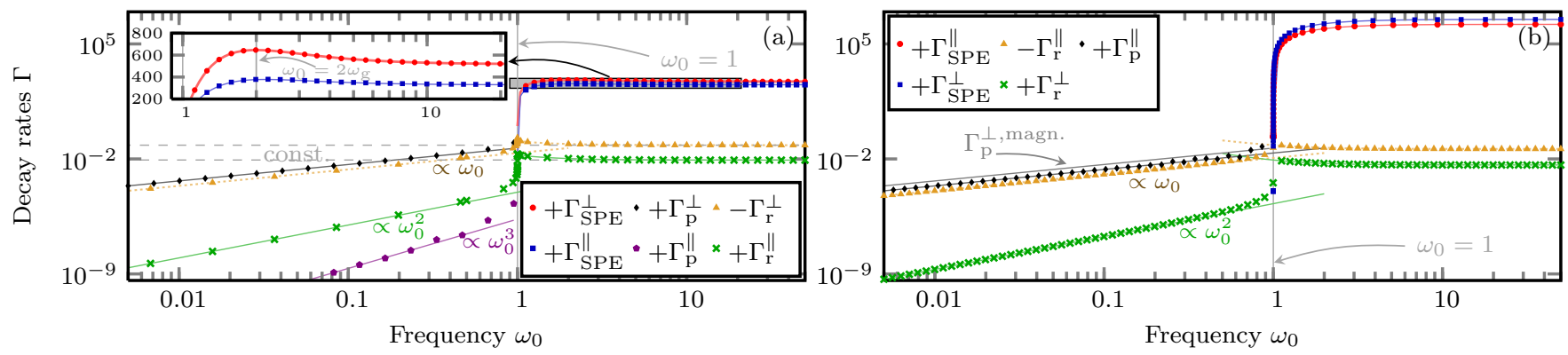

FIG. 4. Orthogonal and parallel decay rates of an emitter situated at $d=\left(3 \times 10^{8}\right)^{-1}$ above a graphene layer suspended in air. The lines represent the analytical approximations discussed in the text while the dots represent numerical results. (a) Results for a magnetic emitter. (b) Results for an electric emitter. Note that the results are plotted on a logarithmic scale so that contributions which lead to an enhancement ( + ) or a suppression ( - ) of the decay rate are signs as indicated by corresponding signs in the insets of panels (a) and (b).

quite abruptly as the frequency of the emitter moves across the band gap and, for larger frequencies, takes on a weakly frequency-dependent value around $\alpha \pi /\left(4 v_{\mathrm{F}} \omega_{\mathrm{g}}^{3} \varepsilon_{m}^{3 / 2}\right) \approx 10^{3}$. In both $\Gamma^{\|, \perp}$, the TE contributions are dominant and lead to the nonmonotonic behavior discussed above.

Most of the above-described characteristics also qualitatively apply to the case of an electric dipole emitter [see Fig. 4(b)]. Indeed, the relevant expressions can be easily obtained by swapping the reflection coefficients in Eq. (4) $[30,32]$. For brevity we will only mention that, as a consequence of the replacement $r^{\mathrm{TM}} \leftrightarrow r^{\mathrm{TE}}$, some features are found in $\Gamma^{\|}$instead of $\Gamma^{\perp}$. Curiously, for $\omega_{0}<\omega_{g}$, due to the proximity of the TE plasmon dispersion relation to the light cone, its contribution to the decay rate is of the same order of magnitude for both emitters and for all distances, i.e., $\Gamma_{\mathrm{p}}^{\mathrm{el}} \approx 2 \Gamma_{\mathrm{p}}^{\text {magn. }} / \sqrt{\varepsilon_{m}}$. More importantly, the SPE channel still provides a large enhancement of the decay rate for $\omega_{0}>1$, featuring again a quite abrupt jump for frequencies near the electronic band gap of graphene. However, for the electric emitter both $\Gamma^{\|, \perp}$ exhibit a monotonous frequency dependence.

In conclusion, the above results suggest atomic or atomlike emitters as sensitive quantum probes to determine the physical properties of graphene and, in particular, to investigate a band gap in its electronic band structure. Using these systems allows for an accurate analysis of this quantity, especially in complex (but relevant for graphene-based technologies) situations where it is no longer spatially homogenous: This occurs, e.g., when the sheet (i) is exposed to mechanical stress [33], (ii) is positioned on an inhomogeneous substrate, or (iii) absorbs impurities (in a controlled [34] or uncontrolled fashion). In our approach, the emitter noninvasively probes graphene's properties in different physical regimes, enabling experimental investigation of unusual graphene properties such as TE surface resonances (see also $[12,13]$ ) and providing results complementary to those accessible when using other procedures. In addition, the possibility to engineer different internal quantum states of the emitter and study their lifetimes can also offer new opportunities which are presently not accessible with other techniques. As a concrete experimental approach, we suggest extending the known use of microtrapped Bose-Einstein condensates [35,36] to map the local band gap structure of graphene sheets with micron resolution. One would detect the spin-flip rate by measuring the spatially dependent spin population after a known time since its preparation as a spin-polarized gas. For enhanced sensitivity, it will be advantageous to employ an optical dipole trap, ideally configured as a light sheet, tuned to a frequency below the main atomic transition. Fluorescence imaging following selective resonant excitation of the emitter decay target state will enable the measurement of even very slow decay rates down to a few events per time across the ensemble of typically $10^{5}$ atoms. The high temporal resolution of this technique can offer an important advantage in analyzing the different (relatively slow) processes cited above.

In addition to atomic quantum gases other very well-suited candidates are Si and NV centers in nanodiamonds. They do not only show tunable magnetic and electric transitions from the $\mathrm{MHz}$ to the $\mathrm{THz}$ frequency range but also simultaneously allow for high position resolution [37]. Small band gaps can be investigated by cooling the system to the $\mathrm{mK}$ regime, such that magnetically tunable Zeeman [38] or hyperfine transitions [39] can be utilized. Our work can open additional pathways to enhance the fundamental understanding of the validity of different graphene models $[24,40,41]$ and also provides relevant information for realistic applications and designs of interest, e.g., in atom-chip research [42-44]. Indeed, this material with its intrinsic, room-temperature quantum properties $[2,5,45]$ has been deemed as a particularly interesting addition to these systems in order to proceed further on the road to quantum computing $[46,47]$.

\section{ACKNOWLEDGMENTS}

We thank Ch. Koller, M. T. Greenaway, and T. M. Fromhold for stimulating discussions. We acknowledge support by the Deutsche Forschungsgemeinschaft (DFG) through the Collaborative Research Center (CRC) 951 "Hybrid Inoganic/Organic Systems for Optoelectronics (HIOS)" within Project No. B10. P.K. acknowledges support from EPSRC (grant EP/K03460X). F.I. further acknowledges financial support from the European Union Marie Curie People program through the Career Integration Grant No. PCIG14- GA-2013-631571 and from the DFG through the DIP program (FO 703/2-1). 
[1] A. K. Geim and K. S. Novoselov, Nat. Mater. 6, 183 (2007).

[2] A. K. Geim, Science 324, 1530 (2009).

[3] A. H. Castro Neto, F. Guinea, N. M. R. Peres, K. S. Novoselov, and A. K. Geim, Rev. Mod. Phys. 81, 109 (2009).

[4] M.-H. Bae, Z. Li, Z. Aksamija, P. N. Martin, F. Xiong, Z.-Y. Ong, I. Knezevic, and E. Pop, Nat. Commun. 4, 1734 (2013).

[5] K. S. Novoselov, Z. Jiang, Y. Zhang, S. V. Morozov, H. L. Stormer, U. Zeitler, J. C. Maan, G. S. Boebinger, P. Kim, and A. K. Geim, Science 315, 1379 (2007).

[6] A. A. Balandin, Nat. Mater. 10, 569 (2011).

[7] B. Wunsch, T. Stauber, F. Sols, and F. Guinea, New J. Phys. 8, 318 (2006).

[8] B. Guo, L. Fang, B. Zhang, and J. R. Gong, Insciences J. 1, 80 (2011).

[9] E. H. Hwang and S. DasSarma, Phys. Rev. B 75, 205418 (2007).

[10] M. Bordag and I. G. Pirozhenko, Phys. Rev. B 89, 035421 (2014).

[11] M. Bordag and I. G. Pirozhenko, Phys. Rev. D 91, 085038 (2015).

[12] G. Gómez-Santos and T. Stauber, Phys. Rev. B 84, 165438 (2011).

[13] A. Y. Nikitin, F. Guinea, F. J. Garcia-Vidal, and L. MartinMoreno, Phys. Rev. B 84, 195446 (2011).

[14] W. J. M. Kort-Kamp, B. Amorim, G. Bastos, F. A. Pinheiro, F. S. S. Rosa, N. M. R. Peres, and C. Farina, Phys. Rev. B 92, 205415 (2015).

[15] K. J. Tielrooij, L. Orona, A. Ferrier, M. Badioli, G. Navickaite, and S. Coop, Nat. Phys. 11, 281 (2015).

[16] G. Giovannetti, P. A. Khomyakov, G. Brocks, P. J. Kelly, and J. van den Brink, Phys. Rev. B 76, 073103 (2007).

[17] J. Jung, A. M. DaSilva, A. H. MacDonald, and S. Adam, Nat. Commun. 6, 6308 (2015).

[18] K.-K. Bai, Y. C. Wei, J. B. Qiao, S. Y. Li, L. J. Yin, W. Yan, J. C. Nie, and L. He, Phys. Rev. B 92, 121405 (2015).

[19] A. Kumar, A. Nemilentsau, K. H. Kin Hung, G. Hanson, N. X. Fang, and T. Low, Phys. Rev. B 93, 041413(R) (2016).

[20] I. V. Fialkovsky, V. N. Marachevsky, and D. V. Vassilevich, Phys. Rev. B 84, 035446 (2011).

[21] M. Chaichian, G. L. Klimchitskaya, V. M. Mostepanenko, and A. Tureanu, Phys. Rev. A 86, 012515 (2012).

[22] R. Nair, P. Blake, A. N. Grigorenko, K. S. Novoselov, T. J. Booth, T. Stauber, N. M. R. Peres, and A. K. Geim, Science 320, 1308 (2008).

[23] S. A. Mikhailov and K. Ziegler, Phys. Rev. Lett. 99, 016803 (2007).

[24] T. Stauber, J. Phys. Condens. Matter 26, 123201 (2014).

[25] J. F. M. Werra, F. Intravaia, and K. Busch, J. Opt. 18, 034001 (2016).

[26] R. J. Glauber and M. Lewenstein, Phys. Rev. A 43, 467 (1991).
[27] F. Intravaia and K. Busch, Phys. Rev. A 91, 053836 (2015).

[28] S. Scheel, L. Knöll, and D.-G. Welsch, Phys. Rev. A 60, 4094 (1999).

[29] P. de Vries and A. Lagendijk, Phys. Rev. Lett. 81, 1381 (1998).

[30] C. Henkel, S. Pötting, and M. Wilkens, Appl. Phys. B 69, 379 (1999).

[31] L. Novotny and B. Hecht, Principles of Nano-optics, 2nd ed. (Cambridge University Press, New York, 2012).

[32] F. Intravaia, C. Henkel, and M. Antezza, in Casimir Physics, Lecture Notes in Physics Vol. 834, edited by D. Dalvit, P. Milonni, D. Roberts, and F. da Rosa (Springer, Berlin, 2011), pp. 345-391.

[33] S. Zhu, J. A. Stroscio, and T. Li, Phys. Rev. Lett. 115, 245501 (2015).

[34] D. C. Elias, R. R. Nair, T. M. G. Mohiuddin, S. V. Morozov, P. Blake, M. P. Halsall, A. C. Ferrari, D. W. Boukhvalov, M. I. Katsnelson, A. K. Geim, and K. S. Novoselov, Science 323, 610 (2009).

[35] S. Wildermuth, S. Hofferberth, I. Lesanovsky, E. Haller, L. M. Andersson, L. Mauritz, and S. Groth, I. Bar-Joseph, P. Krüger, and J. Schmiedmayer, Nature (London) 435, 440 (2005).

[36] S. Wildermuth, S. Hofferberth, I. Lesanovsky, S. Groth, P. Krüger, J. Schmiedmayer, and I. Bar-Joseph, Appl. Phys. Lett. 88, 264103 (2006).

[37] A. W. Schell, P. Engel, J. F. M. Werra, C. Wolff, K. Busch, and O. Benson, Nano Lett. 14, 2623 (2014).

[38] R. Amsüss, Ch. Koller, T. Nöbauer, S. Putz, S. Rotter, K. Sandner, S. Schneider, M. Schramböck, G. Steinhauser, H. Ritsch, J. Schmiedmayer, and J. Majer, Phys. Rev. Lett. 107, 060502 (2011).

[39] A. Tkalčec, S. Probst, D. Rieger, H. Rotzinger, S. Wünsch, N. Kukharchyk, A. D. Wieck, M. Siegel, A. V. Ustinov, and P. Bushev, Phys. Rev. B 90, 075112 (2014).

[40] R. Roldán, J.-N. Fuchs, and M. Goerbig, Solid State Commun. 175, 114 (2013).

[41] D. Brida, A. Tomadin, C. Manzoni, Y. J. Kim, A. Lombardo, S. Milana, R. R. Nair, K. S. Novoselov, A. C. Ferrari, G. Cerullo, and M. Polini, Nat. Commun. 4, 1987 (2013).

[42] R. Folman, P. Krüger, J. Schmiedmayer, J. H. Denschlag, and C. Henkel, Adv. At. Mol. Opt. Phys. 48, 263 (2002).

[43] J. Fortágh and C. Zimmermann, Rev. Mod. Phys. 79, 235 (2007).

[44] G. Sinuco-León, B. Kaczmarek, P. Krüger, and T. M. Fromhold, Phys. Rev. A 83, 021401 (2011).

[45] N. Tombros, C. Jozsa, M. Popinciuc, H. T. Jonkman, and B. J. Van Wees, Nature (London) 448, 571 (2007).

[46] B. Trauzettel, D. V. Bulaev, D. Loss, and G. Burkard, Nat. Phys. 3, 192 (2007).

[47] G.-P. Guo, Z.-R. Lin, T. Tu, G. Cao, X.-P. Li, and G.-C. Guo, New J. Phys. 11, 123005 (2009). 